\title{
Development and simulated validation of a food-frequency questionnaire for the Colombian population
}

\author{
Leonelo E Bautista ${ }^{1, *}$, Oscar F Herrán ${ }^{2}$ and Jane A Pryer ${ }^{3}$ \\ 'Department of Population Health Sciences, University of Wisconsin Medical School, 610 Walnut Street, 703 WARF, \\ Madison, WI 53726-2397, USA: ${ }^{2}$ Centro de Investigación Epidemiológica, Observatorio Epidemiológico de \\ Enfermedades Cardiovasculares, Universidad Industrial de Santander, Bucaramanga, Colombia: ${ }^{3}$ Royal Free \\ and University College Medical School, University College London, Department of Primary Care and Population \\ Sciences, UK
}

Submitted 17 May 2004: Accepted 6 September 2004

\begin{abstract}
Objective: To develop a food-frequency questionnaire (FFQ) useful for ranking of nutrient intakes.

Design: Subjects consuming their regular diet completed 7 days of weighed intake registry (7-WIR). Foods for the FFQ were selected by stepwise multiple regression. The FFQ was then completed for each subject using data on individual food consumption from the 7-WIR. The correlation and agreement between the extrapolated FFQ and the 7-WIR data were assessed using Spearman's rank correlation coefficients $\left(r_{\mathrm{S}}\right)$ and Bland and Altman's limits of agreement (LOA).

Setting: Bucaramanga, Colombia.

Subjects: We studied 97 randomly selected 20-40-year-old subjects.

Results: Sixty foods were selected for the FFQ. The 7-WIR and the extrapolated FFQ intake estimates correlated well. $r_{\mathrm{S}}$ was 0.58 for energy, 0.53 for carbohydrate, 0.50 for total fat, and 0.48 for protein. For micronutrients, $r_{\mathrm{S}}$ varied from 0.46 (manganese) to 0.71 (vitamin $\mathrm{B}_{12}$ ). FFQ average intake estimates were $83 \%, 80 \%, 86.2 \%$ and $86.4 \%$ of 7-WIR estimates for energy, carbohydrate, total fat and protein, respectively. LOA for these nutrients ranged between $45 \%$ and $165 \%$. FFQ micronutrient intakes were on average $96 \%$ (median) of those from the 7-WIR, and the median lower and upper LOA were $50 \%$ and $203 \%$. However, there was no indication that the degree of agreement varied with the level of intake.

Conclusions: According to our simulated validation, this FFQ may be useful to rank subjects by nutrient intake. Its validity against standard independent measurements and its applicability to other subsets of the Colombian population should be carefully considered.
\end{abstract}

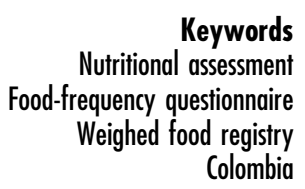

Keywords equency questionnaire Colombia
Dietary factors have a large impact on chronic disease morbidity and mortality ${ }^{1}$. There are few studies on the relationship between nutritional intake and the risk of chronic diseases in developing countries. In Colombia, as in other countries ${ }^{2}$, this is due mainly to the lack of valid, low-cost dietary assessment methods amenable for studies in large populations. Use of dietary tools developed in other populations is not a proper alternative, since it is well known that methods validated in one population are not necessarily accurate in other groups, even from the same country, unless they are developed specifically for this purpose $\mathrm{p}^{3}$. Moreover, the use of methods with low validity seriously attenuates the associations between nutritional intake and disease in epidemiological studies, a problem known as regression dilution ${ }^{4}$.
Although different methods can be used to evaluate individuals' nutritional intake in epidemiological studies, food-frequency questionnaires (FFQs) have been the tool used most frequently during past decades, because they are easy to apply in large populations and have relatively low cost ${ }^{5}$. An FFQ for a specific population is usually developed by modifying existing FFQs validated in a related population. Such an approach was not feasible for our population of interest, since no FFQ had previously been developed in Colombia. Here we report the results of a study aimed to develop an FFQ based on the direct quantification of foods and nutrient intake in a sample from the population of Bucaramanga, Colombia. The FFQ includes the full range of nutrients and may be used in epidemiological studies to rank individuals by their level of nutrient intake. 


\section{Methods}

The study was developed in five phases:

1. Selection of participants;

2. Completion of 7-day weighed intake registry (7-WIR);

3. Review and completion of food composition tables and calculation of individual cumulative nutrient intakes;

4. Selection of a reduced number of foods to be included in the FFQ;

5. Design of the FFQ, completion of the FFQ using the data on food consumption from the 7-WIR, and evaluation of agreement between nutrient intakes estimated from the extrapolated FFQ and the observed 7-WIR.

\section{Selection of participants}

Residents in Bucaramanga, a mid-sized city of approximately 1 million inhabitants in Colombia, were our target population. We studied 97 subjects between 20 and 40 years old who were not on a weight-reducing or any other type of diet. This age group was considered the most appropriate because chronic diseases such as cancer and cardiovascular disease occur mostly after age 50, partly as a consequence of dietary exposure a few decades before. Only subjects who could read and write were included in the study, due to the need to complete a registry of weighed intake. All participants gave their written informed consent. The study was approved by the institutional review board of the School of Medicine, Universidad Industrial de Santander.

Participants were selected by multistage random sampling. First, all city blocks were classified into six socio-economic strata ${ }^{6}$ and five blocks were randomly selected from each stratum. The maps from the 30 blocks selected were updated and 3-4 household units were selected at random from each block. All eligible subjects in each household were identified and listed, and one of them was selected randomly to participate. If the person refused to participate, another eligible subject from the same household was randomly selected.

\section{Weighed intake registry}

All participants completed a registry of foods consumed during seven consecutive days (7-WIR). Before the start of the 7-WIR, a nutritionist trained each participant on how to weigh solid foods, how to measure the volume of liquid foods, and how to complete the registry. All participants were provided with a calibrated scale (sensitivity $0.001 \mathrm{~g}$ ), a calibrated vase (sensitivity $10 \mathrm{~cm}^{3}$ ) and written instructions for completion of the 7-WIR. Following their training, each participant completed a 3-day testing period aimed to evaluate his/her ability to produce complete, good-quality records. On the first day of the training period, each subject completed a questionnaire on age, education, income and occupation.
During the 7-WIR a nutritionist visited each participant at least once, to review the completion of the registry and answer any concerns or doubts from the participant. Participants were able and encouraged to contact the nutritionist at any time they considered it necessary during the period of the 7-WIR. To evaluate significant dietary changes related to study participation, each participant was weighed at the beginning and end of the 7-WIR period.

Finally, during the 7-WIR period, the person in charge of preparing the foods in the household completed a registry with the name of each dish, the type and weight or volume of the ingredients, and the way to cook it. Data on food type and frequency of consumption were used in a later step by the authors to retrospectively complete FFQs for each participant.

\section{Review of food composition tables and calculation of cumulative nutrient intakes}

Three food composition tables were used to calculate individual nutrient intakes ${ }^{7-9}$. The nutritional content of manufactured foods was ascertained from the food label or by questioning the manufacturer. Dishes that were not listed in any of the food composition tables were grouped by name and by ingredients. This grouping was conducted independently by two nutritionists and the disagreements were solved by consensus. Then, we calculated the average content of the ingredients in each dish, using the data collected in the 7-WIR. The nutritional content of these 'typical dishes' was calculated using the food composition tables. To make the calculation of nutritional intake easier, we prepared a new food composition table that included the foods listed in the original tables ${ }^{7-9}$, the manufactured foods and the typical dishes ${ }^{10}$.

The cumulative intake of each nutrient for an individual was calculated, using FoodCalc ${ }^{11}$, as the sum of the product of the amount of food consumed times the nutrient content.

\section{Selection of foods to be included in the FFQ}

To select foods for inclusion in the FFQ, we evaluated their contribution to the inter-individual variability in the intake of each specific nutrient. For this purpose, we ran a series of forward stepwise multiple regression models with the specific nutrient as the dependent variable and the foods consumed as independent variables. Foods with a significance level $<0.10$ were added to the regression model. Stepwise regression identifies those foods that best contribute to discriminate among individuals with different levels of consumption of a given nutrient ${ }^{12}$. This strategy is likely most appropriate, since FFQs are used to classify subjects by their nutrient consumption, preserving their ordered relationship as correctly as possible.

Those foods that contributed significantly to the intake of at least one nutrient of interest were included in the 
FFQ. Foods that did not discriminate between subjects with different levels of intake of a nutrient were excluded, even if they were an important source of that nutrient ${ }^{2}$. Foods that were consumed rarely and those that contributed less than 5\% of the absolute intake of any nutrient were excluded from the stepwise regression.

\section{Design of the FFQ and simulated validation study: evaluation of agreement between retrospectively extrapolated FFQ data and 7-WIR nutrient intakes}

Our FFQ included the foods selected in the previous step and was designed following the recommendations of Willett $^{2}$. Questions about usual food consumption were referred to the previous year. Related foods were grouped within the same section of the FFQ, and those with similar nutritional content were grouped under the same name (e.g. fruit juice), to reduce the number of items in the FFQ. Nine mutually exclusive frequency categories, from 'never' to 'two or more times a day' were used, but portion sizes were not included in the FFQ.

We completed the designed FFQ for each individual, using data on food type and frequency of consumption obtained from the 7-WIR. Then we re-estimated the individual's nutritional intake from these 'extrapolated FFQs', using FoodCalc ${ }^{11}$. To make these calculations, the amount of food consumed was replaced by the mean and by the median of the portion sizes estimated from the 7-WIR data for the whole population, and for age and sex groups. We selected the portion sizes (means or medians) that resulted in a better correlation between the observed nutrient consumption and that estimated by projecting the 7-WIR data into the FFQ. These portion sizes were then integrated in a computer program designed to input the FFQ data and to calculate the individual nutrient intakes in one step (NutCalc ${ }^{\circledR}$ version 1.2) ${ }^{13}$.

\section{Statistical analysis}

Geometric means were used to estimate daily average nutrient intake. The relative agreement of the extrapolated FFQ compared with the 7-WIR was assessed using Pearson correlation coefficients, after transformation to the natural log scale, to take into account that the distribution of most nutrients was positively skewed. We also estimated the non-parametric Spearman rank correlation coefficient using the original data (before $\log _{\mathrm{e}}$ transformation). The Spearman rank correlation coefficient uses the relative order of the nutrient intake and is therefore less sensitive to extreme values than the Pearson coefficient. Correlation coefficients are useful to estimate reliability when nutrient intake is used as a continuous variable in epidemiological studies. However, the Pearson and Spearman correlation coefficients only measure the precision of the relationship between the FFQ and the 7-WIR. To measure both the precision and the accuracy of the relationship we used Lin's concordance correlation coefficient ${ }^{14,15}$. Lin's coefficient evaluates whether the observed data deviate significantly from the line of perfect concordance (i.e. the line at $45^{\circ}$ ). If a scatter plot defined by FFQ and 7-WIR values is very tight (precision) and very close to the line of perfect concordance (accuracy), then Lin's coefficient will increase towards a maximum value of -1 or +1 . Correlation coefficients do not provide an appropriate evaluation of relative validity when dietary data are used in categories or groups in the analysis of epidemiological studies ${ }^{16}$. To evaluate the performance of the extrapolated FFQ in categorising subjects into the same group as the 7-WIR, we calculated Cohen's weighted kappa statistic $^{17,18}$. The weighted kappa corrects for chance agreement in rank scales and takes into account the magnitude of the disagreement by giving less weight to larger discrepancies. Quartile values of the observed distribution of extrapolated FFQ and 7-WIR nutrient intakes were used as cut points for cross-tabulation, with weights equal to $1-|i-j| /(k-1)$, where $i$ and $j$ index the rows and columns of the table and $k=4$ (the maximum number of possible ratings).

Correlation coefficients have been criticised as a measure of agreement because they measure the strength of a linear relationship between two variables, but give no information about the magnitude and direction of the difference (absolute bias) between the two variables ${ }^{19,20}$. To examine the actual agreement between the extrapolated FFQ and the 7-WIR, we used the limits of agreement (LOA) method proposed by Bland and Altman ${ }^{19,20}$. Finally, we fit a linear regression with the absolute bias as the dependent variable and the mean of the FFQ and 7WIR as the independent variable to evaluate if the bias changed significantly with magnitude of intake ${ }^{21}$. This analysis was conducted with the $\log _{\mathrm{e}}$-transformed data, and absolute bias and LOA were exponentiated and multiplied by 100 to express the relative increase in extrapolated FFQ compared with 7-WIR measured intake.

\section{Results}

\section{Studied population}

A total of 97 subjects (14-17 from each socio-economic stratum) participated in the study. The average age was 28 years, 45 (46.4\%) were women and 61\% were married (Table 1 ). Only $6 \%$ had not completed primary school, but $61 \%$ had an educational level above high school. These sociodemographic characteristics were similar in men and women. The average body weight was $66.1 \mathrm{~kg}(95 \%$ confidence interval (CI) 63.2-68.9) at the beginning and $66.0 \mathrm{~kg}(95 \% \mathrm{CI}$ 63.2-69.8) at the end of the 7-WIR $(P=0.758)$.

\section{Weighed intake registry}

A total of 115 households with eligible subjects were visited. One eligible subject was selected at random from each household. Only 97 volunteers (84.3\%) were willing to participate in the 3-day testing period and capable of 
Table 1 Sociodemographic characteristics of the studied population sample. Data are expressed as $n(\%)$

\begin{tabular}{lccc}
\hline Characteristic & Men & Women & All \\
\hline Socio-economic status* & & & \\
1 & $8(15.4)$ & $8(17.8)$ & $16(16.5)$ \\
2 & $8(15.4)$ & $9(20.0)$ & $17(17.5)$ \\
3 & $9(17.3)$ & $7(15.6)$ & $16(16.5)$ \\
4 & $9(17.3)$ & $8(17.8)$ & $17(17.5)$ \\
5 & $9(17.3)$ & $8(17.8)$ & $17(17.5)$ \\
6 & $9(17.3)$ & $5(11.1)$ & $14(14.4)$ \\
Age (years) & $26(50.0)$ & $16(35.6)$ & $42(43.3)$ \\
$20-24$ & $9(17.3)$ & $11(24.4)$ & $20(20.6)$ \\
$25-29$ & $8(15.4)$ & $3(6.7)$ & $11(11.3)$ \\
$30-34$ & $9(17.3)$ & $15(33.3)$ & $24(24.7)$ \\
$35-40$ & $17(32.7)$ & $21(46.7)$ & $38(39.2)$ \\
Marital status & $35(67.3)$ & $24(53.3)$ & $59(60.8)$ \\
Single & $3(5.8)$ & $3(5.8)$ & $6(6.2)$ \\
Married & $20(38.5)$ & $12(26.7)$ & $32(33.0)$ \\
Educational level & $29(55.8)$ & $30(66.7)$ & $59(60.8)$ \\
Primary school or less & & & \\
High school & &
\end{tabular}

${ }^{*}$ Higher values correspond to higher socio-economic status; see reference 6 .

producing accurate records. They were included in the study and none of them dropped out during the 7 days of weighted intake registry. Some $39.2 \%$ of subjects lost 91 of the 2037 expected regular meals during the week (4.5\%), but the pattern of missing meals was not related to gender, age, socio-economic status or level of education. A total of 7810 records (one per consumed food item) were gathered. Of the records, $21.4 \%$ were for foods consumed during breakfast, 39.5\% during lunch, 26.0\% during dinner and $13.1 \%$ in other meals.

\section{Review of food composition tables and calculation of cumulative nutrient intakes}

The 7810 records of the 7-WIR corresponded to 310 different foods. Out of these, 214 were complex food preparations or dishes. The average content of ingredients for each dish was estimated using the recipes obtained during the 7-WIR. For a group of 35 dishes prepared in restaurants, the average content of ingredients was obtained from cookery books. In addition, the nutritional content of five foods was obtained directly from the manufacturer. Once the average content of ingredients in complex preparations was ascertained, we calculated their nutrient content, as well as that of single food items, using different food composition tables ${ }^{7-9}$. Complex preparations and manufactured foods were then incorporated in a new food composition table ${ }^{10}$.

On average, during the 7-WIR, the participants consumed $8339 \mathrm{~kJ}$ (1992 kcal), $67 \mathrm{~g}$ protein, $287 \mathrm{~g}$ carbohydrate and $64 \mathrm{~g}$ fat daily (Table 2). As expected, daily intake was significantly greater in men than in women for all nutrients, except for dietary fibre $(P=0.11)$, iron $(P=0.10)$ and manganese $(P=0.78)$. On average (median), men consumed 36\% more energy and macronutrients, and 36\% more micronutrients, than did women.

\section{Selection of foods to be included in the FFQ}

The number of foods selected by stepwise regression varied from four for iron to 46 for carbohydrate (Table 3). The proportion of the variability in intake of macronutrients explained by the regression models $\left(R^{2}\right)$ varied from $59 \%$ for polyunsaturated fat to $99 \%$ for carbohydrate. For only two micronutrients, iron and manganese, was $R^{2}$ below $50 \%$. Taken as a whole, 60 out of 310 different foods consumed by the participants were identified for inclusion in the FFQ. For macronutrients, a regression model with the 60 foods included in the FFQ explained from $62 \%$ of the variability in individual intake $\left(R^{2}\right)$ of polyunsaturated fat to $96 \%$ of the variability for total energy. For micronutrients, only the $R^{2}$ for manganese was below 50\% (Table 3).

\section{Design of the FFQ and simulated validation study}

The 60 foods selected for inclusion in the FFQ were organised into 14 mutually exclusive groups according to their similarity: meats, fried foods, eggs, whole grains, refined grains, dairy products, sauces, desserts, sugared drinks, legumes, fruits, fresh vegetables, cooked vegetables and alcoholic drinks. Overall means and medians for portion sizes resulted in practically identical estimations of nutrient consumption to those from use of means and medians specific for age, gender and socioeconomic status.

Nutrient intakes estimated from the FFQ completed using 7-WIR data on food consumption were compared with those estimated from the 7-WIR data. For macronutrients, the Pearson correlation coefficient $(R)$ varied from 0.52 for protein to 0.57 for carbohydrate (Table 4), while for all micronutrients it was greater than 0.50 , with the exception of iron (0.28), manganese (0.29) and vitamin $\mathrm{B}_{6}$ (0.48). A similar pattern was observed with the concordance correlation coefficient $\left(\rho_{\mathrm{c}}\right)$, but the coefficients for carbohydrate $(0.45)$, protein $(0.47)$, crude fibre (0.49), sodium (0.46) and vitamin $\mathrm{B}_{6}(0.45)$ were slightly smaller than 0.50 . On the other hand, the correlation between ordered values of nutrient intakes, estimated with the Spearman rank correlation coefficient $\left(r_{\mathrm{S}}\right)$, was 0.58 for energy, 0.53 for carbohydrate, 0.50 for total fat and 0.48 for protein. For micronutrients, $r_{\mathrm{S}}$ varied from 0.46 (manganese) to 0.71 (vitamin $\mathrm{B}_{12}$ ). The weighted agreement beyond chance (kappa) for a classification in four groups ranged from 0.33 (total fat) to 0.38 (protein) for macronutrients and energy and from 0.28 (crude fibre) to 0.56 (cholesterol) for micronutrients.

The extrapolated FFQ underestimated the daily intakes of energy and all macronutrients (Table 4). Energy intake estimated from the FFQ was roughly $83 \%$ of that estimated from the 7-WIR, but in 95\% of subjects these estimates were between $49 \%$ and $139 \%$ of the 7 -WIR estimates. 
Table 2 Average daily nutrient intakes estimated from the 7-day weighed intake registry. Data are expressed as mean ( $95 \%$ confidence interval)

\begin{tabular}{|c|c|c|c|}
\hline Nutrient & Total $(n=97)$ & $\operatorname{Men}(n=52)$ & Women $(n=45)$ \\
\hline \multicolumn{4}{|c|}{ Energy and macronutrients } \\
\hline Energy $(\mathrm{kJ})$ & $8339.3(7808.6-8906.1)$ & $9671.3(8917.8-10488.9)$ & $7026.6(6461.5-7641.1)$ \\
\hline Carbohydrate (g) & $286.8(268.5-306.5)$ & $329.1(302.1-358.6)$ & $244.7(225.2-265.9)$ \\
\hline \multicolumn{4}{|l|}{ Fat $(\mathrm{g})$} \\
\hline Total & $64.2(59.6-69.1)$ & $74.2(67.9-81.1)$ & $54.2(48.8-60.2)$ \\
\hline Monounsaturated & $18.0(16.6-19.5)$ & $21.2(19.1-23.4)$ & $14.9(13.4-16.6)$ \\
\hline Polyunsaturated & $8.2(7.5-9.0)$ & $8.9(8.1-9.9)$ & $7.4(6.4-8.6)$ \\
\hline Saturated & $20.1(18.6-21.7)$ & $23.3(21.2-25.5)$ & $17.0(15.2-19.0)$ \\
\hline Protein $(\mathrm{g})$ & $66.9(62.4-71.7)$ & $75.9(69.6-82.7)$ & $57.8(52.5-63.7)$ \\
\hline \multicolumn{4}{|l|}{ Micronutrients } \\
\hline Calcium (mg) & $514.8(475.5-557.3)$ & $568.9(517.1-625.9)$ & $458.6(404.1-520.4)$ \\
\hline Cholesterol (mg) & 259.7 (232.5-290.2) & $298.2(254.5-349.5)$ & 221.4 (191.3-256.2) \\
\hline Crude fibre $(\mathrm{g})$ & $5.4(5.0-5.8)$ & $6.1(5.4-6.8)$ & $4.7(4.2-5.3)$ \\
\hline Copper $(\mathrm{mg})$ & $0.7(0.7-0.8)$ & $0.9(0.8-1.0)$ & $0.6(0.5-0.7)$ \\
\hline Dietary fibre (g) & $2.6(2.3-2.9)$ & $2.8(2.4-3.3)$ & $2.3(1.9-2.8)$ \\
\hline Folic acid $(\mu \mathrm{g})$ & $192.7(176.3-210.6)$ & $235.8(213.6-260.4)$ & $152.6(134.6-172.9)$ \\
\hline Iron $(\mathrm{mg})$ & $15.1(13.4-16.9)$ & $16.5(14.7-18.5)$ & $13.6(11.0-16.8)$ \\
\hline Magnesium (mg) & $169.4(158.1-181.6)$ & $192.7(176.8-210.1)$ & $146.0(132.4-160.9)$ \\
\hline Manganese (mg) & $1.0(0.8-1.1)$ & $1.0(0.9-1.1)$ & $0.9(0.7-1.3)$ \\
\hline Niacin (mg) & $14.5(13.4-15.7)$ & $16.9(15.3-18.7)$ & $12.1(11.0-13.4)$ \\
\hline Pantothenic acid (mg) & $3.0(2.8-3.3)$ & $3.5(3.2-3.8)$ & $2.6(2.4-2.9)$ \\
\hline Phosphorus (mg) & $1044.1(976.7-1116.1)$ & $1205.0(1111.3-1306.7)$ & $884.7(809.0-967.4)$ \\
\hline Potassium (mg) & $2132.4(1990.0-2285.0)$ & 2443.5 (2241.9-2663.3) & $1821.9(1658.0-2002.0)$ \\
\hline Riboflavin (mg) & $1.3(1.2-1.4)$ & $1.5(1.3-1.7)$ & $1.1(1.0-1.2)$ \\
\hline Sodium (mg) & $779.7(691.2-879.6)$ & $943.1(803.8-1106.6)$ & $625.8(530.0-739.0)$ \\
\hline Thiamine (mg) & $1.0(0.9-1.1)$ & $1.1(1.0-1.3)$ & $0.8(0.7-0.9)$ \\
\hline Vitamin A (RE) & $654.8(588.6-728.6)$ & 779.1 (672.4-902.7) & $535.7(466.8-614.8)$ \\
\hline Vitamin $B_{6}(\mathrm{mg})$ & $1.3(1.2-1.4)$ & $1.4(1.3-1.6)$ & $1.1(1.0-1.3)$ \\
\hline Vitamin $B_{12}(\mu \mathrm{g})$ & $4.2(3.7-4.7)$ & $5.2(4.5-6.1)$ & $3.2(2.8-3.7)$ \\
\hline Vitamin C (mg) & $124.5(113.2-136.9)$ & $143.7(125.8-164.0)$ & $105.5(93.2-119.4)$ \\
\hline Zinc (mg) & $7.6(7.0-8.2)$ & $9.1(8.2-10.1)$ & $6.2(5.6-6.8)$ \\
\hline
\end{tabular}

$\mathrm{RE}$ - retinol equivalents.

All $P$-values for the gender comparison were $<0.05$, with the exception of polyunsaturated fats $(P=0.30)$, dietary fibre $(P=0.06)$, iron $(P=0.58)$, manganese $(P=0.151)$ and vitamin $\mathrm{B}_{6}(P=0.399)$.

Results for carbohydrate were very similar to those for energy intake. For total fat and protein intakes the extrapolated FFQ estimates were $86 \%$ of those from the 7WIR, but for any randomly selected subject the FFQ estimates are expected to be between $45 \%$ and $163 \%$ of 7 WIR estimates. However, there was no indication that the relative biases for macronutrients varied across the range of intake ( $P$-values from 0.36 to 0.83 ).

Extrapolated FFQ intake estimates for micronutrients were on average $96 \%$ (median) of those from the 7-WIR (Table 4). The average FFQ intake for 16 out of 22 micronutrients was between $90 \%$ and $110 \%$ of the 7 -WIR estimates, and only one was below 80\% (sodium) and two above $110 \%$ (insoluble dietary fibre and vitamin $\mathrm{B}_{12}$ ). Overall, the median lower and upper LOA for all micronutrients were $50 \%$ and $203 \%$ (from half to twice the average of the 7-WIR estimates). However, considerable variability was observed among micronutrients. For example, for manganese the intake could be grossly underestimated (20\%) or overestimated (456\%) in 95\% of the subjects, while for phosphorus these figures would only be $51 \%$ to $150 \%$. For 13 out of 22 micronutrients (59\%), the extrapolated FFQ estimates were between half and two times the 7-WIR estimates in 95\% of the subjects. Only the errors for iron $(P=0.00)$, manganese $(P=0.00)$ and niacin $(P=0.03)$ increased significantly with the increase in the mean intake of the nutrient.

\section{Discussion}

The development of nutritional epidemiological research in developing countries with a rising burden of chronic non-infectious diseases is of great public health importance, but has been hampered by the lack of methods amenable for valid measurement of dietary exposure. The majority of FFQs have been developed as adaptations from two existing questionnaires originally devised for use in the US population ${ }^{22}$. Because of the difference in dietary patterns, those questionnaires cannot be adapted for use in the Colombian population. Therefore, we designed an FFQ using data from weighed food records as the starting point for the selection of foods and portion sizes. We found for most nutrients that average intake estimated from the extrapolated FFQs had an acceptable level of agreement and correlation with that from the 7-WIR. Therefore, this FFQ may be useful for epidemiological studies in literate 20-40-year-old subjects from Bucaramanga, and may serve as a basic Colombian questionnaire that can be adapted and changed according to needs. However, issues related to the understanding of 
Table 3 Number of foods selected by stepwise multiple linear regression for each nutrient, and coefficients of determination $\left(R^{2}\right)$ for these models and for a model including 60 foods selected for inclusion in the food-frequency questionnaire

\begin{tabular}{|c|c|c|c|}
\hline Nutrient & $\begin{array}{l}\text { Number } \\
\text { of foods } \\
\text { selected }\end{array}$ & $\begin{array}{c}R^{2} \text { from the } \\
\text { model for } \\
\text { the nutrient }\end{array}$ & $\begin{array}{c}R^{2} \text { from the } \\
\text { model with } \\
60 \text { foods }\end{array}$ \\
\hline \multicolumn{4}{|c|}{ Energy and macronutrients } \\
\hline Energy & 30 & 0.96 & 0.96 \\
\hline Carbohydrate & 46 & 0.99 & 0.94 \\
\hline \multicolumn{4}{|l|}{ Fat } \\
\hline Total & 35 & 0.95 & 0.95 \\
\hline Saturated & 20 & 0.87 & 0.91 \\
\hline Monounsaturated & 28 & 0.95 & 0.97 \\
\hline Polyunsaturated & 14 & 0.59 & 0.62 \\
\hline Protein & 25 & 0.87 & 0.92 \\
\hline \multicolumn{4}{|l|}{ Micronutrients } \\
\hline Calcium & 23 & 0.93 & 0.90 \\
\hline Cholesterol & 18 & 0.94 & 0.95 \\
\hline Crude fibre & 24 & 0.94 & 0.92 \\
\hline Copper & 36 & 0.98 & 0.96 \\
\hline $\begin{array}{l}\text { Dietary fibre, } \\
\text { insoluble }\end{array}$ & 19 & 0.96 & 0.94 \\
\hline Folic acid & 20 & 0.88 & 0.95 \\
\hline Iron & 4 & 0.35 & 0.59 \\
\hline Magnesium & 33 & 0.95 & 0.96 \\
\hline Manganese & 4 & 0.16 & 0.26 \\
\hline Niacin & 27 & 0.94 & 0.96 \\
\hline Pantothenic acid & 32 & 0.95 & 0.96 \\
\hline Phosphorus & 32 & 0.94 & 0.96 \\
\hline Potassium & 36 & 0.97 & 0.96 \\
\hline Riboflavin & 22 & 0.93 & 0.97 \\
\hline Sodium & 23 & 0.97 & 0.98 \\
\hline Thiamine & 28 & 0.95 & 0.97 \\
\hline Vitamin A & 20 & 0.94 & 0.96 \\
\hline Vitamin $\mathrm{B}_{6}$ & 9 & 0.50 & 0.66 \\
\hline Vitamin $B_{12}$ & 23 & 0.98 & 0.98 \\
\hline Vitamin C & 35 & 0.99 & 0.98 \\
\hline Zinc & 30 & 0.97 & 0.98 \\
\hline
\end{tabular}

the questions and completion of the questionnaire may affect the performance of the FFQ in large epidemiological studies, and should be the subject of future research.

The development and validation of an FFQ is a difficult task, due in part to the difficulties in obtaining a sample representative of the population to which the FFQ will be applied. Because of its design, our study could only include subjects able to read and write and those who were willing and capable of completing the dietary records accurately. Within this group we selected a random sample of subjects from different socio-economic strata. Although we used a narrow age range, we cannot verify whether the age distribution of our sample was similar to that in the general population because of a lack of updated demographic data. On the other hand, the proportion of married subjects in the general population $(39.2 \%)$ is similar to that in the target population $(34.0 \%)^{23}$. The level of education was likely higher in our sample. Therefore, the validity of the FFQ may be lower if administered to subjects with lower level of education, even though the fraction of subjects with primary school or less in the general population (6.2\%) was close to that in the target population $(4.7 \%)^{23}$.
Foods selected for inclusion in the FFQ explained more than $90 \%$ of the inter-individual intake variability for all nutrients with the exception of iron, manganese and vitamin $\mathrm{B}_{6}$. This approach allowed us to select the best set of foods while accounting for possible correlations between different foods ${ }^{2,12}$. Considerable effort was made to update the available Colombian food composition tables, to minimise errors related to nutrient contents. The estimation of the average contents of complex food preparations and their inclusion in an updated food composition table ${ }^{10}$ facilitated the calculation of nutrient contents from the diet records and the FFQ, while improving the accuracy of our estimates. In addition, weighed food records were completed for 7 days in an attempt to design an FFQ able to capture day-to-day variability.

Because of their specific limitations, we used different types of correlation measurements to evaluate the agreement between nutrient intakes estimated with the FFQ and with the 7-WIR. Although the correlation coefficient has been questioned as an indicator of validity, because it measures association instead of actual agreement between two measurement methods ${ }^{20,24}$, its use in our analysis can be justified in as far as the FFQ was designed to rank individuals according to nutrient intake instead of quantifying their absolute level of intake ${ }^{16}$. Pearson correlation coefficients close to 0.60 have been considered an indicator of good validity ${ }^{24}$. In our study they were above 0.60 for 17 of 28 nutrients and below 0.50 only for iron, manganese and vitamin $\mathrm{B}_{6}$. These values are similar to those reported in previous validation studies in Latin American populations ${ }^{25,26}$. Appropriate concordance between the extrapolated FFQ data and the 7-WIR was also shown by the Spearman rank correlation coefficient, which is less sensitive to extreme values than the Pearson coefficient. Gross misclassification is unlikely when Spearman coefficients are $\geq 0.50^{16}$, and only three nutrients from the extrapolated FFQ had coefficients below that value (crude fibre, iron and manganese). Moreover, for most nutrients, the concordance correlation coefficients showed substantial correlation between FFQ and 7-WIR estimates. This indicates considerable agreement between the two methods, even when the systematic differences in the estimates from both methods (bias) are taken into account in the calculation of the correlation coefficient.

The high correlation between extrapolated FFQ and 7-WIR estimates suggests that dietary data from the FFQ could be used as a continuous variable in the analysis of epidemiological studies. However, some degree of attenuation in the estimates of relative risk should be expected. This should be taken into account in the design of epidemiological studies. Increasing the sample size or conducting parallel calibration studies may help to reduce the impact of the added random error originating from the FFQ estimates ${ }^{2}$.

The weighted kappa statistic may be a more appropriate option to evaluate FFQ performance if nutrient intake is to 
Table 4 Correlation, concordance and bias between individual intakes of nutrients as measured with the 7-day weighed intake registry and the extrapolated food-frequency questionnaire

\begin{tabular}{|c|c|c|c|c|c|c|}
\hline Nutrient & $R^{\star}$ & $\rho_{\mathrm{c}} \dagger$ & $r_{\mathrm{S}} \ddagger$ & Kappa§ & Bias (LOA) (\%) & $\begin{array}{c}\text { Linear } \\
\text { trend } P \text {-value }\end{array}$ \\
\hline \multicolumn{7}{|c|}{ Energy and macronutrients } \\
\hline Energy & 0.61 & 0.51 & 0.58 & 0.36 & $82.9(49.3-139.2)$ & 0.36 \\
\hline Carbohydrate & 0.57 & 0.45 & 0.53 & 0.34 & $79.9(46.3-137.9)$ & 0.60 \\
\hline \multicolumn{7}{|l|}{ Fat } \\
\hline Total & 0.57 & 0.52 & 0.50 & 0.33 & $86.2(45.6-162.7)$ & 0.59 \\
\hline Monounsaturated & 0.64 & 0.63 & 0.59 & 0.44 & $94.7(51.0-176.1)$ & 0.42 \\
\hline Polyunsaturated & 0.57 & 0.50 & 0.62 & 0.41 & $79.8(36.8-173.2)$ & 0.27 \\
\hline Saturated & 0.59 & 0.58 & 0.53 & 0.33 & $92.9(50.0-172.5)$ & 0.41 \\
\hline Protein & 0.52 & 0.47 & 0.48 & 0.38 & $86.4(45.3-164.7)$ & 0.83 \\
\hline \multicolumn{7}{|l|}{ Micronutrients } \\
\hline Calcium & 0.73 & 0.68 & 0.70 & 0.49 & 85.7 (48.7-151.0) & 0.73 \\
\hline Cholesterol & 0.72 & 0.72 & 0.70 & 0.56 & $101.0(47.8-213.4)$ & 0.20 \\
\hline Crude fibre & 0.50 & 0.49 & 0.43 & 0.28 & $105.0(46.9-234.9)$ & 0.94 \\
\hline Copper & 0.64 & 0.64 & 0.57 & 0.37 & $102.0(54.6-189.3)$ & 0.63 \\
\hline Dietary fibre, insoluble & 0.62 & 0.58 & 0.68 & 0.44 & $125.0(45.4-345.6)$ & 0.77 \\
\hline Folic acid & 0.66 & 0.65 & 0.63 & 0.41 & $96.6(45.0-207.3)$ & 0.08 \\
\hline Iron & 0.28 & 0.22 & 0.45 & 0.34 & $83.4(27.6-251.9)$ & 0.00 \\
\hline Magnesium & 0.65 & 0.64 & 0.60 & 0.39 & $95.4(55.4-164.2)$ & 0.33 \\
\hline Manganese & 0.29 & 0.22 & 0.46 & 0.33 & $94.3(19.5-456.3)$ & 0.00 \\
\hline Niacin & 0.65 & 0.64 & 0.67 & 0.39 & $100.0(56.0-178.8)$ & 0.03 \\
\hline Pantothenic acid & 0.65 & 0.64 & 0.57 & 0.38 & $102.0(55.9-173.3)$ & 0.72 \\
\hline Phosphorus & 0.63 & 0.57 & 0.61 & 0.36 & $87.6(51.4-149.5)$ & 0.77 \\
\hline Potassium & 0.64 & 0.63 & 0.61 & 0.36 & $96.3(55.7-167.0)$ & 0.39 \\
\hline Riboflavin & 0.66 & 0.64 & 0.64 & 0.42 & $110.0(60.4-199.0)$ & 0.10 \\
\hline Sodium & 0.62 & 0.46 & 0.64 & 0.49 & $62.9(24.3-163.2)$ & 0.77 \\
\hline Thiamine & 0.65 & 0.64 & 0.59 & 0.38 & $104.0(58.3-186.5)$ & 0.99 \\
\hline Vitamin A & 0.68 & 0.68 & 0.65 & 0.46 & $104.0(48.2-223.2)$ & 0.10 \\
\hline Vitamin $\mathrm{B}_{6}$ & 0.48 & 0.45 & 0.55 & 0.39 & $95.1(41.8-216.6)$ & 0.00 \\
\hline Vitamin $\mathrm{B}_{12}$ & 0.69 & 0.68 & 0.71 & 0.47 & $111.0(50.6-244.0)$ & 0.46 \\
\hline Vitamin $\mathrm{C}$ & 0.56 & 0.55 & 0.53 & 0.36 & $104.0(43.7-246.9)$ & 0.20 \\
\hline Zinc & 0.64 & 0.64 & 0.62 & 0.41 & $96.8(52.5-178.2)$ & 0.39 \\
\hline
\end{tabular}

${ }^{*}$ Pearson correlation coefficient.

† Lin's concordance correlation coefficient ${ }^{14,15}$

¥ Spearman correlation coefficient.

$\S$ Cohen's weighted kappa statistic for quartiles (weight $=1-|i-j| /(k-1)$, where $i$ and $j$ index the rows and columns of the two ratings and $k=4$ ).

ๆ Relative individual intake difference and limits of agreement ${ }^{20}$.

be categorised during the analysis of epidemiological studies. Kappa values showed fair agreement (0.21-0.40) for 17 and moderate agreement $(0.41-60)$ for 11 nutrients. However, cross-classification using quartiles may group subjects with very different intakes into the same category and may misclassify subjects with similar intakes if they are close to the cut-off point. Therefore, our results suggest that, in using FFQ intake estimates as exposure in epidemiological studies, one should carefully evaluate whether to group subjects instead of using nutrient intake as a continuous variable, and how such groups should be created in order to minimise misclassification error.

The extrapolated FFQ average intake estimates were very close those from the 7-WIR, but the LOA were wide. Only $59 \%$ of the nutrients were within the $50 \%$ under- to $100 \%$ overestimation suggested by Ambrosini et al. as acceptable $^{21}$. This indicates that the use of the FFQ could lead to considerable under- or overestimation of individual average intake for some nutrients. However, the FFQ could be useful to quantify differences in mean intake across populations and over time. Moreover, since the biases in the mean intake estimates for most nutrients were unrelated to the average mean intake, it is reasonable to expect that no differential bias will occur in the study of diet-disease relationships ${ }^{24}$. Therefore, mostly nondifferential misclassification and attenuation of the relative risk would result from the use of the FFQ. Increasing sample size and conducting calibration studies may help in dealing with the FFQ-associated non-differential error in epidemiological studies ${ }^{2,27}$.

We acknowledge that a main limitation of our study is that the comparison of extrapolated FFQ and 7-WIR nutrient intakes does not constitute a proper evaluation of the validity of the FFQ, because the extrapolated FFQs were completed using the data on food frequency consumption from the 7-WIR. Although this eliminated errors related to imperfect recall and selective reporting of food, the concordance between the extrapolated FFQ and the 7-WIR is likely overestimated, and does not reflect the actual validity of an FFQ completed by study subjects. Had the concordance between the extrapolated FFQ and the 7-WIR been poor, we would have concluded that the FFQ could not provide a valid measurement of nutrient intake, even in the best scenario. However, the fact that 
the extrapolated FFQ and the 7-WIR correlated well suggests that the FFQ may indeed be useful in the measurement of nutrient intake and warrants a more rigorous evaluation of validity. Also, we did not explore whether the performance of the FFQ was dependent on individual characteristics such as sex, education and obesity, because of sample size limitations. If errors were dependent on such characteristics, differential misclassification in the measurement of intake could occur and could lead to distortions other than attenuation of the relative risk in epidemiological studies. An evaluation of the validity of the FFQ in an independent sample of the target population is currently underway.

Our study shows that this FFQ may be useful as a dietary assessment tool for future epidemiological studies in the population of Bucaramanga. However, careful consideration should be given to issues related to study design in order to control for non-differential measurement error of nutrient intake. This FFQ could also be used as a starting point for the development and validation of FFQs in other populations from Colombia, and to monitor changes across populations and through time.

\section{Acknowledgements}

Contributions: All authors participated in the conceptualisation and design of the study. L.E.B. overviewed the conduction of the study, carried out most of the data analysis and wrote the manuscript. O.F.H. was in charge of the fieldwork, participated in the analysis of the data, designed the questionnaire and reviewed the manuscript. J.A.P. collaborated in the interpretation of data results and reviewed the final manuscript.

Sponsorship: The work was funded by the Instituto Colombiano para el Desarrollo de la Ciencia y la Tecnología 'Francisco José de Caldas' (COLCIENCIAS), Contract No. 126-2002; code 1102-04-11719.

\section{References}

1 Bowen DJ, Beresford SA. Dietary interventions to prevent disease. Annual Review of Public Health 2002; 23: 255-86.

2 Willett WC, ed. Food-frequency methods. In: Nutritional Epidemiology. New York: Oxford University Press, 1998; 3-17.

3 Kristal AR, Feng Z, Coates RJ, Oberman A, George V. Associations of race/ethnicity, education, and dietary intervention with the validity and reliability of a food frequency questionnaire: the Women's Health Trial Feasibility Study in Minority Populations. American Journal of Epidemiology 1997; 146: 856-69.

4 Day N, McKeown N, Wong M, Welch A, Bingham S. Epidemiological assessment of diet: a comparison of a 7-day diary with a food frequency questionnaire using urinary markers of nitrogen, potassium and sodium. International Journal of Epidemiology 2001; 30: 309-17.

5 Prentice RL. Dietary assessment and the reliability of nutritional epidemiology reports. Lancet 2003; 362: 182-3.

6 Departamento Nacional de Planeación (DNP). Estratificación Socioeconómica. Manual General. Cabeceras
Municipales Tipo 3 y Localidades o Centros Poblados Hasta con Tres Mil Habitantes. Bogotá, Colombia: DNP, 1994.

7 US Department of Agriculture. Nutritive Value of Food. Washington, DC: Government Printing Office, 1988.

8 Instituto Colombiano de Bienestar Familiar. Tabla de Composición de Alimentos Colombianos, 6th ed. Santa Fe de Bogotá, Colombia: Ministerio de Salud, 1992.

9 Centro de Atención Nutricional. Tabla de Composición de Alimentos. Medellin, Colombia: Centro de Atención Nutricional, 1990

10 Herran O, Bautista L. Tabla de Composición de Alimentos Consumidos en Bucaramanga. Bucaramanga, Colombia: Universidad Industrial de Santander, 2002.

11 Lauritsen J. FoodCalc 1.3: Diet, Cancer and Health project at the Danish Cancer Society, Copenhagen, Denmark, 1998

12 Mark SD, Thomas DG, Decarli A. Measurement of exposure to nutrients: an approach to the selection of informative foods. American Journal of Epidemiology 1996; 143: 514-21.

13 Bautista L, Herrán-Falla O. NutCalc [1.2]. Bucaramanga, Colombia: Universidad Industrial de Santander, 2001.

14 Lin LI-K. A concordance correlation coefficient to evaluate reproducibility. Biometrics 1989; 45: 255-68.

15 Lin LI-K. A note on the concordance correlation coefficient. Biometrics 2000; 56: 324-5.

16 Masson LF, McNeill G, Tomany JO, Simpson JA, Peace HS, Wei L, et al. Statistical approaches for assessing the relative validity of a food-frequency questionnaire: use of correlation coefficients and the kappa statistic. Public Health Nutrition 2003; 6: 313-21.

17 Cohen J. A coefficient of agreement for nominal scales. Educational and Psychological Measurement 1960; 20: $37-46$.

18 Cohen J. Weighted kappa: nominal scale agreement with a provision for scaled disagreement or partial credit. Psychological Bulletin 1968; 70: 213-20.

19 Bland JM, Altman DG. Measuring agreement in method comparison studies. Statistical Methods in Medical Research 1999; 8: $135-60$.

20 Bland JM, Altman DG. Statistical methods for assessing agreement between two methods of clinical measurement Lancet 1986; 1: 307-10.

21 Ambrosini GL, van Roosbroeck SA, Mackerras D, Fritschi L, de Klerk NH, Musk AW. The reliability of ten-year dietary recall: implications for cancer research. Journal of Nutrition 2003; 133: 2663-8.

22 Cade J, Thompson R, Burley V, Warm D. Development, validation and utilisation of food-frequency questionnaires - a review. Public Health Nutrition 2002; 5: 567-87.

23 PROFAMILIA. Encuesta Nacional de Demografia y Salud. Resumen Región Oriental. Bogotá, Colombia: PROFAMILIA, 2000.

24 Ambrosini GL, de Klerk NH, Musk AW, Mackerras D. Agreement between a brief food frequency questionnaire and diet records using two statistical methods. Public Health Nutrition 2001; 4: 255-64.

25 Navarro A, Osella AR, Guerra V, Munoz SE, Lantieri MJ, Eynard AR. Reproducibility and validity of a food-frequency questionnaire in assessing dietary intakes and food habits in epidemiological cancer studies in Argentina. Journal of Experimental \& Clinical Cancer Research 2001; 20: 365-70.

26 Slater B, Philippi ST, Fisberg RM, Latorre MR. Validation of a semi-quantitative adolescent food frequency questionnaire applied at a public school in Sao Paulo, Brazil. European Journal of Clinical Nutrition 2003; 57: 629-35.

27 Johansson I, Hallmans G, Wikman A, Biessy C, Riboli E, Kaaks R. Validation and calibration of food-frequency questionnaire measurements in the Northern Sweden Health and Disease cohort. Public Health Nutrition 2002; 5: 487-96. 